\title{
Spatially and temporally resolved measurements of argon metastable atoms in the effluent of a cold atmospheric pressure plasma jet
}

\author{
R. Bussiahn, E. Kindel, H. Lange and K.-D. Weltmann \\ Leibniz Institute for Plasma Science and Technology (INP), Felix-Hausdorff-Str. 2, \\ 17489 Greifswald, Germany \\ E-mail: bussiahn@inp-greifswald.de
}

\begin{abstract}
Although atmospheric pressure plasma jets (APPJ) have been used for several years in wide fields of application, there is still lack of knowledge about physical aspects, namely the phenomenon of spatiotemporally localized luminous effects in the effluent of APPJ, so called "plasma bullets".

The present paper reports on investigations on the effluent of a low frequency (kHzrange) driven APPJ in argon atmosphere. To gain insight into the spatiotemporal structure of the effluent, laser absorption measurements, probing the optical depth of the $4 s^{3} P_{2}-4 p{ }^{3} D_{3}$ transition of argon $(811.531 \mathrm{~nm}$ ), which is proportional to the column density of the metastable $4 s^{3} P_{2}$ argon atoms and investigations with an intensified video camera were performed. Simultaneous recordings of the discharge current show that plasma bullets are connected only to a certain current pulse in a series of four pulses of the discharge cycle. A good correlation between the propagation speeds of the plasma bullets $(5-20 \mathrm{~km} / \mathrm{s})$ and of the generation zone of metastable argon atoms in the effluent of the APPJ $(8-25 \mathrm{~km} / \mathrm{s})$ was found. Both, the appearance of plasma bullets and spatiotemporal evolution of the generation zone of metastable atoms can be explained by the effect of a self-propagating ionization front.
\end{abstract}

PACS numbers: 52.50.Dg, 52.80.Hc 


\section{Introduction}

Over the past years non-thermal atmospheric pressure plasma jets have attracted noticeable interest for surface modification techniques. A wide range of applications came up, as plasma activation of surfaces for adhesion control, surface cleaning, deposition of functional coatings or biomedical appliances. Some recent reports [1-8] deal with the temporal and spatial development - mostly driven in helium- of APPJ effluents. For the experimental conditions discussed there, it has been shown, that the plasma in the effluent is formed by fast-moving plasma "bullets" with velocities of more than $10 \mathrm{~km} / \mathrm{s}$. This value exceeds the gas velocity by three orders of magnitude. Presently two explanations for this phenomenon exist. The authors of [2] suggest a streamer model based on photoionization to explain the effect. Shi et al. [4] argue that the dielectric barrier discharge forms a hypersonic plasma bullet train due to an ionization wave. In the present work, the simultaneous existence of plasma bullets and metastable argon atoms outside of an APPJ, operated with argon gas flow into ambient air is reported.

\section{Experimental setup}

A schematic diagram of the plasma source used in this study is shown in figure 1a and has been described in detail in [9]. It consist of a central stainless steel rod electrode (1 $\mathrm{mm}$ diameter) inside of a quartz capillary (outer diameter $4 \mathrm{~mm}$, inner diameter 1.6 $\mathrm{mm}$ ) and a grounded slit electrode, which permits to observe the discharge gap. The inner electrode is powered by a sinusoidal amplifier/generator $(10 \mathrm{kHz}-20 \mathrm{M} \mathrm{Hz}, \mathrm{AG}$ 1021, T\&C Power Conversion, Inc.) in connection with a HV-transformer at a frequency of $40 \mathrm{kHz}$ and voltages of $3-5 \mathrm{kV}$. The Ar gas flow rate was set to $0.5 \mathrm{slm}$ (gas velocity $4 \mathrm{~m} / \mathrm{s}$ ) to get laminar conditions.

The apparatus used for laser atom absorption spectroscopy (LAAS) is shown schematically in figure 2 . The method has already proven its principle applicability under conditions of very short absorption lengths (tenths of millimeter) in measurements on single filaments of dielectric barrier discharges [10]. Here, the wavelength of an external cavity diode laser (Littman configuration, Sacher Lasertechnik) was tuned to the peak absorption of the $4 s^{3} P_{2}-4{ }^{3} D_{3}$ transition of $\operatorname{argon}(811.531 \mathrm{~nm})$ and the temporal behavior of the transmission (or absorbance) was measured. Basically, LAAS allows the determination of absolute particle number densities of excited species. Nevertheless, in case of our experimental conditions, where we had to deal with very small, variable and not exactly known absorption lengths and inhomogeneously distributed absorbers in the line of sight, only measurements of relative values of the optical depth of the named transition were possible. Furthermore, a quantitative analysis would require the knowledge of the absorption line profile function of the transition. As the line profile function of the transition is extended over $60 \mathrm{pm}$, it was not possible to measured it completely with our laser system. 
The laser beam was divided into three parts by two beam splitters (BS). One part was directed side-on to the effluent of the APPJ and its intensity was measured with a fast photodiode (PD). The jet was located on a two-dimensional stage that can be translated in vertical and horizontal direction, perpendicular to the laser beam. A second part was lead to a wavemeter (Burleigh WA-10, $1 \mathrm{pm}$ spectral resolution) to control the wavelength of the laser. The third beam part entered the plasma of a low pressure argon reference discharge and was used to regulate the laser wavelength exactly to the maximum value of the absorption line profile. Before the laser beam was focused onto the effluent with a lens (L), the intensity was attenuated by a pinhole and neutral density filters to avoid saturation of the optical transition. An interference filter (IF) between plasma and photodiode reduced the contribution of the plasma radiation to the signal intensity. Another lens focused the laser beam to the photodiode. The use of a 500 $\Omega$ terminating resistor assured sufficient temporal resolution for proper signal recovery during the pulse phase of the jet. Simultaneously with the transmitted signal a digitizing oscilloscope, utilizing the average function, recorded the electrical characteristics of the jet.

Time resolved photographs of the effluent of the plasma jet were taken by an intensified video camera (4Quik 05A, Stanford Computer Optics) with an exposure time (intensifier gate width) of $40 \mathrm{~ns}$. The delay of the intensifier gate was related to the leading edge of the first current pulse in the anode phase of the discharge. A step size of $40 \mathrm{~ns}$ from picture to picture was chosen to image the emission processes in the effluent.

All measurements have been performed under standard laboratory conditions (room temperature, normal pressure, relative humidity: $40 \% \pm 10 \%)$.

\section{Results and Discussion}

\subsection{Electrical characteristics and absorption signal}

Figure 3 shows typical waveforms of the applied sinusoidal voltage and the total current, which consist of the displacement current and three discharge current pulses $(1-3)$ in the anode phase (powered electrode = anode) and one pulse (4) in the cathode phase. Additionally, the transmission of the laser radiation through the effluent is shown. From these measurements, the absorption signal was derived. This signal is directly proportional to the line-of-sight integrated metastable particle number density or column density. Remarkably, a significant absorption signal exists only during the third current pulse.

The presented electric characteristics depicts a special, well defined mode of operation where plasma bullets are generated. It turned out that the APPJ exhibits certain modes of stability which are correlated with the appearance of distinct current pulses. The discharge ignites at about $2 \mathrm{kVpp}$ where at first a negative current pulse appears (cf. pulse 4 in figure 3). By raising the applied voltage slightly, an additional 
negative current pulse and two positive pulses appear. In an intermediate state, at further increased voltage, even three pulses of positive and negative polarity can be observed. At about $2.6 \mathrm{kVpp}$ the current curve is characterized by a strong, broad, positive pulse (stable in time), followed by a group of stochastically distributed positive pulses (similar to Trichel pulses) and a single negative pulse. With growing voltage the negative pulse gets shifted towards earlier times and the positive pulse splits-up into the pulses 1 and 2 (shown in figure 3), whereas the first pulse can be associated with a pre-discharge and the second one to the main discharge in the interelectrode gap. At about $3.5 \mathrm{kVpp}$ a third structure with positive polarity grows from the group of "Trichel pulses" and evolves into a stable, continuous current pulse which is associated with the formation of plasma bullets. Generally, by raising the driving voltage the observed pulses are shifted towards earlier times.

\subsection{Light emission: active discharge zone vs. plasma bullets}

A photograph of the APPJ, as it appears to the human eye is shown in figure 1b. In this time integrated image (exposure time 1/15 s) the most intensive area of light emission appears in the interelectrode space where the active discharge occurs. Directly at the nozzle exit a bright channel follows, which is surrounded by a pale sheath. Firstly, the diameter of this sheath decreases with increasing distance from the nozzle. At about two-thirds of the visible effluent the sheath and the bright channel are no longer distinguishable from each other. At this point the effluent exhibits its smallest diameter. The last third of the effluent is characterized by a glow which becomes more and more diffuse. An axial cross-section of the effluent shows a constant decrease of the light intensity which is similar to an exponentially decreasing curve. It is seen from figure $1 \mathrm{~b}$ that light emitting species show an inhomogeneous radial distribution. It can be assumed that a similar radial profile applies for metastable atoms.

Figure 4 shows a sequence of images taken for each current pulse in the anode phase at different delay times from the start of the first discharge pulse. At the beginning of the first current pulse (pre-discharge, figure 4(a), 0 ns delay) a plasma bullet starts from the tip of the inner electrode. After $0.08 \mu \mathrm{s}$ two bullets appear whereas the first one travels with a speed of $6 \mathrm{~km} / \mathrm{s}$ out of the capillary and finally disappears in a distance of $1 \mathrm{~mm}$ from the capillary tip. Almost all discharge activity extinguishes after $0.4 \mu \mathrm{s}$. Under the given experimental conditions (ICCD gate width $=40 \mathrm{~ns}$ ) the length of the bullets does not exceed $400 \mu \mathrm{m}$.

In figure 4(b) the discharge activity during the second current pulse (main discharge) is shown. Again, the activity starts at the tip of the inner electrode. Contrary to the pre-discharge phase a strong discharge develops between the two electrodes. No visible plasma exists outside of the capillary.

The behavior during the third current pulse (figure 4(c)) seems to be similar to the results observed for the pre-discharge phase (pulse 1). No visible discharge activity is seen between the electrodes. Seemingly, the outer electrode has no influence on the 
discharge event. A spatiotemporally extended plasma bullet comes off the tip of the inner electrode and leaves the quartz capillary. The whole plasma is more extended and intensive than in case of current pulse 1 , the plasma bullet reaches a length of up to 4 $\mathrm{mm}$ here. Therefore it was possible to get an absorption signal during this current pulse 3. While the plasma bullet propagates along the argon gas channel into the surrounding air, the luminous effect changes its axial extension. During the early stage, when the bullet is located close to the nozzle, the bullet shows an ratio of about 1:10 between diameter and length. The more the plasma departs from the nozzle, the bullet gets compressed in axial direction and finally has a ratio of about 1:2. For the first $240 \mathrm{~ns}$ the evaluation of the images provides a mean propagation velocity of $20 \mathrm{~km} / \mathrm{s}$ and after that time a value of $5 \mathrm{~km} / \mathrm{s}$. The observation that the launch of the spatiotemporally extended bullet is not connected with the current pulse of the main discharge between the electrodes at the leading edge of the driving voltage was also reported by Lu et. al. [2]. Like our observations the release of the plasma bullet is bound to the subsequent current pulse (pulse 3 in our work).

An interesting effect takes place in the end of the plasma bullet phase. At the time $5.00 \mu \mathrm{s}$ in figure $4 \mathrm{c}$ a second plasma bullet appears close to the nozzle exit. The adjacent picture $(5.16 \mu \mathrm{s})$ shows the formation of an additional, weak luminous effect at the end of the first third of the effluent which travels opposite to the direction of gas flow, towards the second plasma bullet $(5.32 \mu \mathrm{s})$.

The 4th pulse (cathode phase of the discharge) is connected with a long lasting diffuse glow at the tip of the inner electrode.

\subsection{Metastable argon atoms: spatiotemporal evolution of relative densities}

Figure 5 describes the axial distribution of the relative metastable column density at different times. The distribution of metastable argon atoms has a spatially extended structure differing from that of the plasma bullet shown in the image sequences. Highest values of the metastable particle number density are always observed in front of the capillary outlet. In the beginning the particle number density decreases linearly with the distance from the nozzle. The position of the tip, where metastable atoms are being created, moves forward by and by. After $200 \mathrm{~ns}$ and $2 \mathrm{~mm}$ from the nozzle a weak maximum of the density appears and travels away from the capillary outlet.

Time integrated measurements of the relative column density of the metastable argon atoms from the nozzle exit to the effluent tip allow to identify the afterglow with three different regions (figure 6). The first is characterized by a decrease of the metastable density. In part, the observed trend may be influenced by a decreasing absorption length in the direction of the gas flow (c.f. discussion on figure $1 \mathrm{~b}$ in section 3.2 for the distribution of radiating species). A nearly constant density is observed from $1 \mathrm{~mm}$ to $3.5 \mathrm{~mm}$ in our experimental conditions. Afterwards the particle number density drops down again up to a distance of $6 \mathrm{~mm}$, which is due to the spatial decay of the metastable atoms. This behavior is similar to line-of-sight integrated measurements 
of the emission intensities in the visible spectral range, which show that the radiating plasma zone extends over a length of $6-8 \mathrm{~mm}$.

The observed structures of the plasma bullets depend on the way how the photographs had been taken and represent a time integrated measurement over the gate width of the ICCD camera. The luminous effect of the bullet is created by a propagating ionization front. The duration of light emission at a certain position is determined by the natural lifetime of the atomic line emission of the argon p-levels. Compared to this the lifetime of metastable argon atoms is significantly higher. Although being created in the same moment as the plasma bullets by the ionization front, metastable atoms decay slower than the light emission from the argon p-levels lasts. Due to this delay the observed distribution of metastable argon atoms gets smeared out over the whole effluent whereas the luminous effect of the plasma bullet is always restricted to a certain part of it. The maximum of metastable particle number density close to the tip of the curves appears because metastable states relax slowly due to quenching processes after being quickly generated due to the effect of the propagating ionization front.

\subsection{Propagation of the ionization front}

The time delay $\Delta \mathrm{t}$ between the leading edge of the third discharge pulse and the peak of the absorbance in dependence of the distance $\mathrm{x}$ from nozzle is presented in figure 7. The curve represents the averaged result of three independent measurements. In a simplified description of the curve, two regions can be defined, which stand for typical mean propagation velocities of the ionization front, creating the metastable states of argon. In the first region, from $0.5 \mathrm{~mm}$ to $3 \mathrm{~mm}$ from the nozzle exit, the slope $\Delta \mathrm{t} / \mathrm{x}$ of a linear approximation of the curve gives a mean velocity of $\sim 20-25 \mathrm{~km} / \mathrm{s}$. In the adjacent part from $3 \mathrm{~mm}$ to $6 \mathrm{~mm}$ a linear fit yields a slope of $\sim 8 \mathrm{~km} / \mathrm{s}$. The given values of the propagation velocity are to be considered as typical, mean values.

The spatial variability of the ionization front propoagation velocity has been found and discussed already for APPJ discharges operated with helium [2,11]. The propagation velocity of the ionization front is strongly determined by the electron drift velocity $\nu_{e}=\mu_{e} E$. The electron mobility $\mu_{e}$ in turn depends on the gas composition. It is clear that the argon gas channel, when it leaves the nozzle exit, intermixes more and more with the surrounding air. Therefore the electron mobility decreases from the nozzle to the tip of the effluent and leads to a reduction of the propagation speed of the ionization front. The influence of the electric field at the head of the plasma bullet is discussed different in literature. Xiong et. al. [11] observe an equivalency between the electron drift velocity and the plasma bullet speed. They assume a high local electric field strength at the bullet head which accelerates electrons towards the bullet head and leads to a self propagating ionization front. On the other hand, Lu and Laroussi [2] consider the case of a weak electric field, which is determined by electrode geometry and applied voltage. The role of the local field at the bullet head is not considered explicitly. For this conditions they propose a streamer propagation model and emphasize the role of 
photoionization prozesses.

Apart from the mechanism, how electrons are generated, whether by photoionization or by collisions, they will be accelerated in the electric field of the ionization front. Collisions of these electrons with neutrals are the chief clause for the population of metastable and resonant excited 1s-states (Paschen notation). The population of the $2 \mathrm{p}$-levels in collisional and radiative processes is very complex. Nevertheless, collisions of neutrals or atoms in the lowest excited states with electrons, gaining their energy in the electric field of the ionization front will play an important role. The 2 p-levels are strongly depopulated to 1s-states in radiative processes. This in turn is observed here in the form of the plasma bullets.

\section{Conclusions}

The phenomenon of spatiotemporally localized luminous effects in the effluent of an APPJ with an argon gas flow into ambient air, so called "plasma bullets" was demonstrated by time resolved photography. It was also shown that metastable argon atoms can be detected under conditions of very short absorption lengths (few tenths of milimeter) in the effluent of an APPJ by means of a laser absorption technique. The results prove the generation of metastable argon atoms in the effluent of the APPJ during a certain discharge phase which is associated with the observation of plasma bullets. Furthermore the corresponding discharge current pulse represents a special mode of stability of the APPJ operation which develops from a series of "Trichel pulses" by increasing the applied voltage to a certain amount.

The time integrated distribution of the column densities of the metastable atoms along the effluent axis is similar to the development of the emission intensities in the visible spectral range.

A good correlation between the propagation velocities of plasma bullets and the shift of the generation zone of metastable argon atoms has been found. This is probably caused by the effect of a propagating ionization front, which simultaneously generates both excited atoms, relaxing in radiative processes (plasma bullets) and atoms in metastable states. The different lifetime of these excited atomic states results in the bullet-like structure in the distribution of radiating atoms and the broad spatio-temporal distribution of atoms in metastable states.

\section{Acknowledgments}

The authors are grateful to P. Holtz and T. Gerling for providing practical assistance.

\section{References}

[1] M Teschke, J Kedzierski, E G Finantu-Dinu, D Korzec, and J Engemann. IEEE Trans. Plasma Sci., 33(2, Part 1):310-311, 2005.

[2] X P Lu and M Laroussi. J. Appl. Phys., 100(6):063302, 2006. 
[3] K Kitano and S Hamaguchi. In Proc. ISPC 18, Kyoto, pages 27A-a5, 2007.

[4] J Shi, F Zhong, J Zhang, D W Liu, and M G Kong. Physics of Plasmas, 15(1):013504, 2008.

[5] J L Walsh and M G Kong. IEEE Trans. Plasma Sci., 36(4, Part 1):954-955, 2008.

[6] B L Sands, B N Ganguly, and K Tachibana. Appl. Phys. Lett., 92(15):151503, 2008.

[7] R Ohyama, M Sakamoto, and A Nagai. J. Phys. D: Appl. Phys., 42(10):105203, 2009.

[8] N Mericam-Bourdet, M Laroussi, A Begum, and E Karakas. J. Phys. D: Appl. Phys., 42(5):055207, 2009.

[9] R Foest, E Kindel, A Ohl, M Stieber, and K-D Weltmann. Plasma Phys. Control. Fusion, 47:B525-B536, 2005.

[10] H Lange and R Bussiahn. In V N Ochkin, editor, Selected Research Papers on Nonequilibrium Plasma at Elevated Pressures, volume 4460 of Proceedings of SPIE, pages 177-187, 2002.

[11] Q Xiong, X Lu, J Liu, Y Xian, Z Xiong, F Zou, C Zou, W Gong, J Hu, K Chen, X Pei, Z Jiang, and Y Pan. J. Appl. Phys., 106:083302, 2009. 


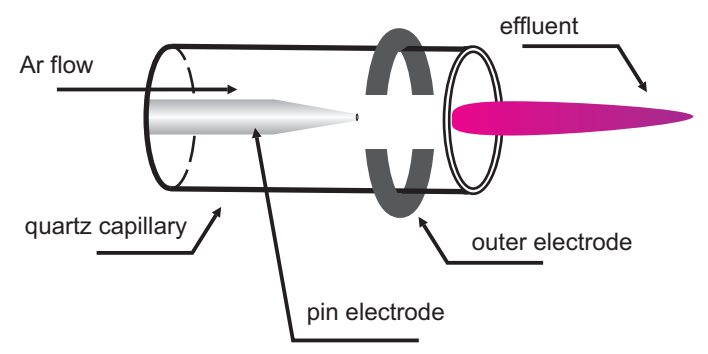

(a)

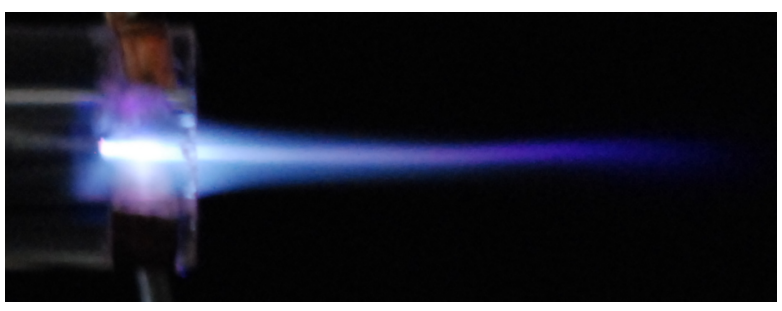

(b)

Figure 1. Cross-sectional view of the APPJ (a) and photograph of the visible plasma emission. (b)

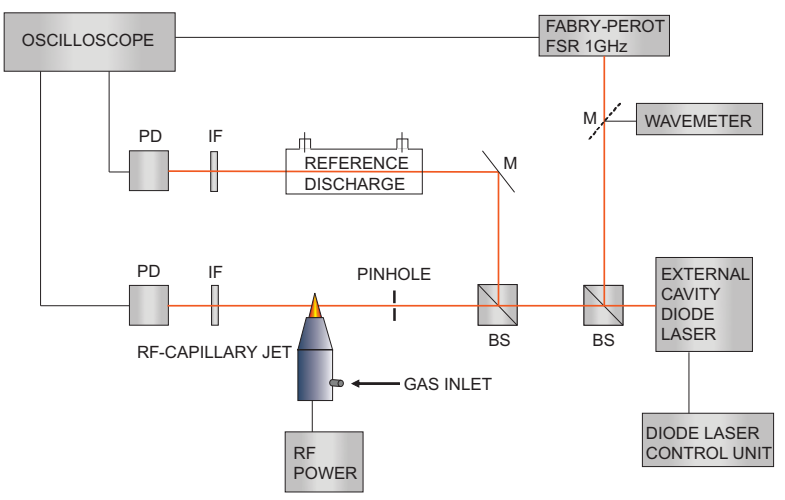

Figure 2. Experimental setup for spatially resolved diode laser absorption measurements. PD-photodiode, IF-interference filter, BS-beamsplitter, M-mirror. 


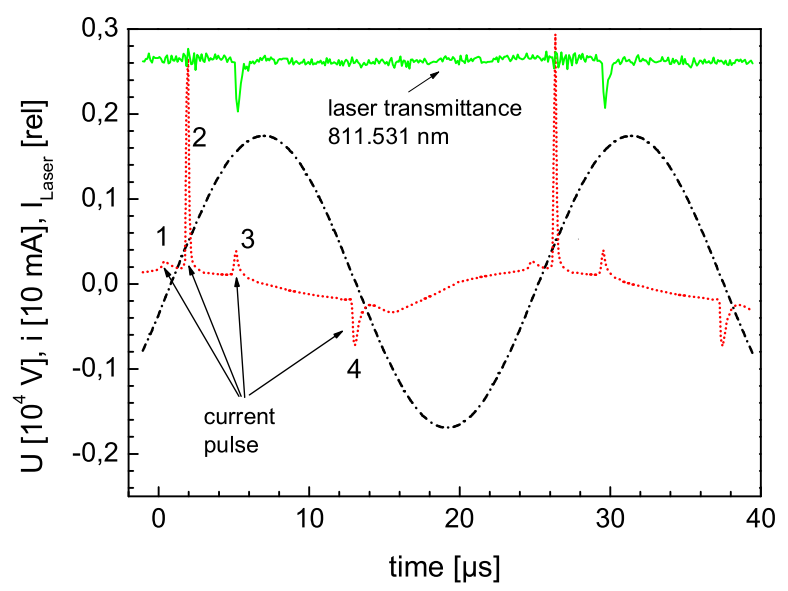

Figure 3. Waveforms of the laser absorption signal, measured $1 \mathrm{~mm}$ in front of the APPJ nozzle (solid line), the applied voltage (dash-dotted line) and total discharge current (dotted line). Arrows indicate three different current peaks in the anode phase and one peak in the cathode phase of the discharge. Further details of the discharge current curve are given in figure 4 . 

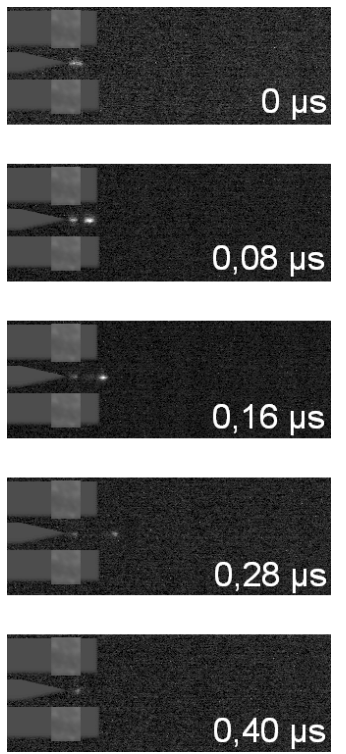

(a)
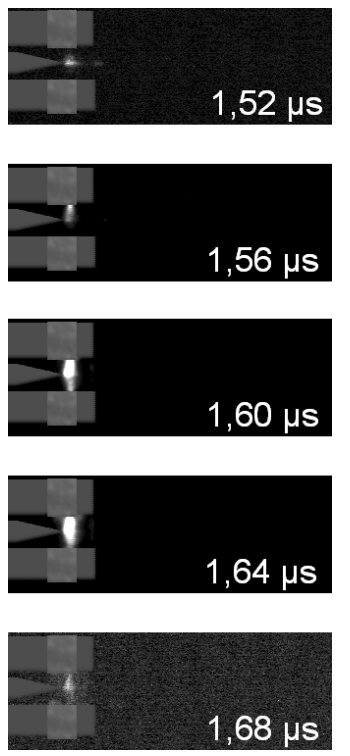

(b)


(c)

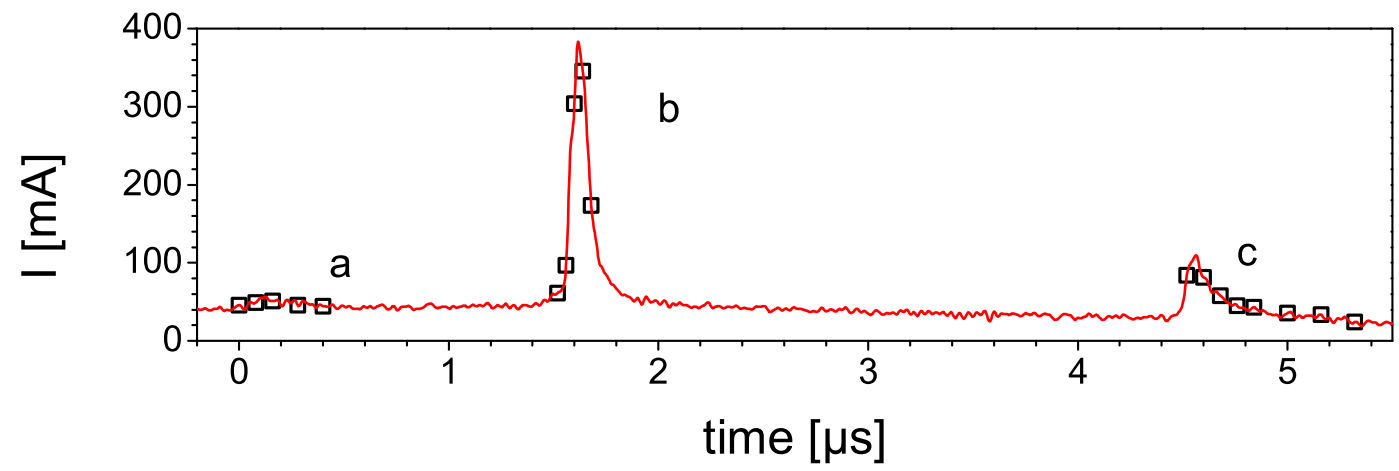

(d)

Figure 4. Short time images (40 ns exposure time) of the plasma emission during the three current pulses in the anode phase of the discharge and the related curve of the total discharge current with open squares, indicating the times to which the figures (a)-(c) are assigned to. (a) pulse 1: pre-discharge connected to the release of a tiny plasma bullet, (b) pulse 2: main discharge where optical emission is limited to the interelectrode gap and (c) pulse 3: release of spatio-temporally extended plasma bullet. 


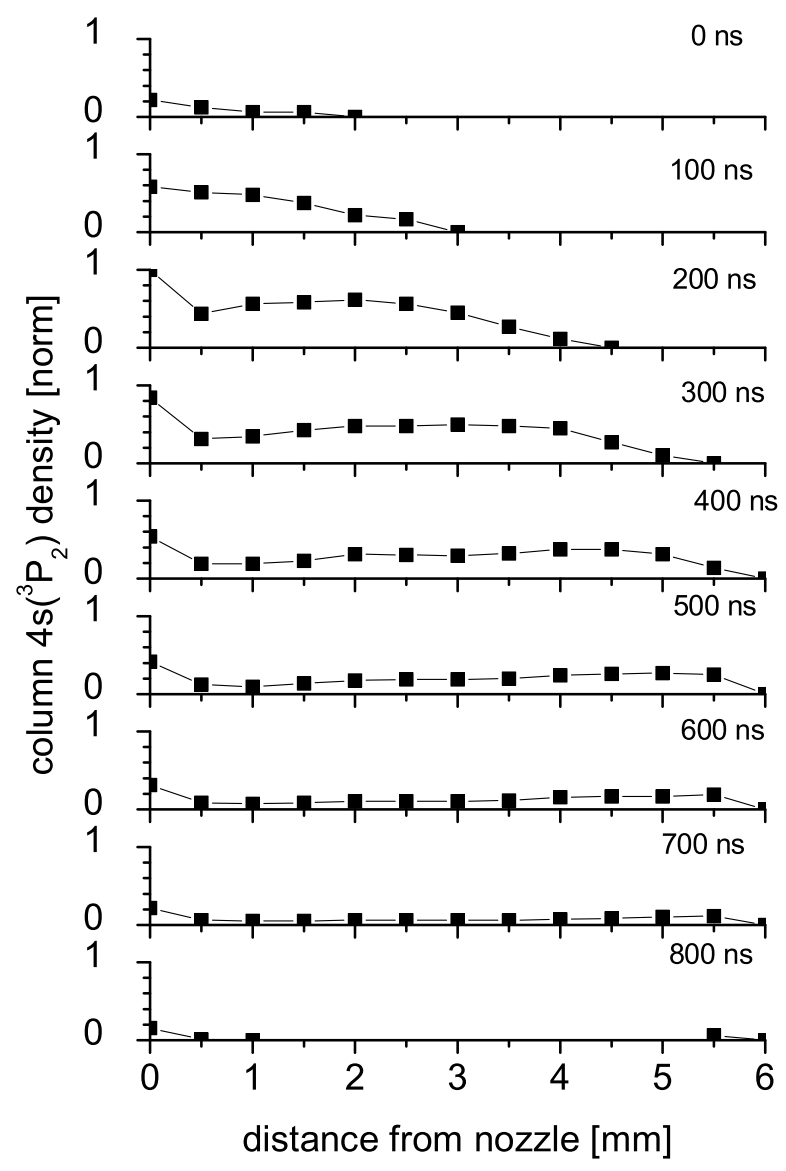

Figure 5. Normalized spatial distribution of the line-of-sight integrated metastable Ar-density at different times from the beginning of the third discharge pulse in the positive half period of the sine wave voltage. Values in each diagram are normalized to the maximum value obtained in this series of measurement $(\mathrm{d}=0 \mathrm{~mm}$ for $\mathrm{t}=200$ ns).



Figure 6. Time integrated relative column density of metastable argon atoms along the effluent axis. Lines are drawn to guide the eyes. 




Figure 7. Time delay between the beginning of the third current pulse and the maximum of the laser absorption as a function of the distance from the nozzle. 